\title{
LE FROMAGE BLANC COMIME ALIMENT EXCLUSIF, OU PRESQUE EXCLUSIF DU RAT BLANC
}

\author{
par

\section{Charles RICHET et Robert MARET}

Plusieurs méthodes permettent d'étudier un aliment. La plus simple consiste à mettre un animal à un régime composé de ce seul aliment. Ce n'est la meilleure que dans quelques cas assez rares. De fait, cette méthode aboutit, le plus souvent, à la mort de l'animal : mort précoce ou mort tardive, avec telle ou telle lésion, tel ou tel trouble. Un aliment, donné comme seule nourriture, est presque toujours diéto-toxique suivant l'expression de MourIQUAND:

Il y a, en effet, soit déséquilibre alimentaire (et on sait les travaux de Mme Randoin, de Lecoq, de LESNÉ et les nôtres sur les perturbations que provoque ce déséquilibre), soit carence de tel ou tel élément (acides a minés, acides gras non saturés, vitamines), infiniment petits chimiques, dont notre organisme ne peut faire la synthèse.

Or, il n'est, croyons-nous, que deux aliments (les seuls qui le soient spécifiquement) qui contiennent tous les éléments indispensables : l'œuf et surtout le lait.

Nous avons étudié le fromage blane comme seul aliment sur le rat blanc (souche du Laboratoire de l'Académie de Médecine).

Ce fromage (1) présehte la composition suivante :

\begin{tabular}{|c|c|}
\hline & 63,65 \\
\hline Acidité totale (en acide lactique) ... & 2,34 \\
\hline Matières grasses . . . . . . . . . . & 15,42 \\
\hline Matièrès azotées ........ & 14,44 \\
\hline 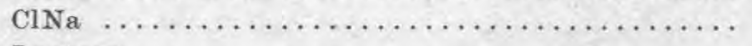 & 1,93 \\
\hline$\ldots \ldots \ldots \ldots \ldots \ldots \ldots \ldots \ldots$ & 2,24 \\
\hline 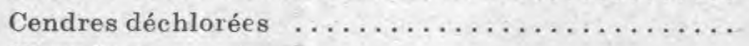 & 1,18 \\
\hline 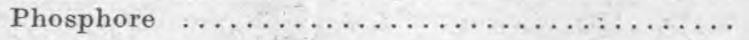 & 0,184 \\
\hline 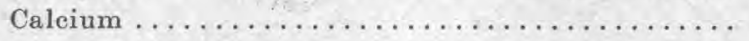 & 0,080 \\
\hline
\end{tabular}

Ce régime est déséquilibré. Il est très pauvre en sucre, trop riche en protides et en lipides (2). De plus il est carencé, car il ne contient que peu de fer et de cuivre.

Au début, trois lots furent constitués - chacun de trois ani-

(1) Nous remercions ici $M$. Corbière de la Maison Gervais qui nous l'a fourni tant pour nos recherches cliniques qu'expérimentales.

(2) Le régime optimum humain doit comprendre, certes, une proportion importante de glycides. Néanmoins, rappelons que des centaines̀ de générations d'esquimaux se sont suecédées, qui n'ont vécu que du produit de leurs pêches et de leur chasse, alimentation très pauvre en glycides. 
maux, deux femelles et un mâle - du poids moyen de 67 grammes, âgés de cinq semaines environ, dans la même eage (on les isolera au moment de la mise-bas).

Le premier lot est mis au fromage seul.

$\mathrm{Au}$ second lot, nous donnerons en plus par animal et par jour 8 grammes de légumes verts crus (choux ou salade) coupés et formant une pâte avec le fromage.

Le troisième lot est mis au régime de base de notre élevage :

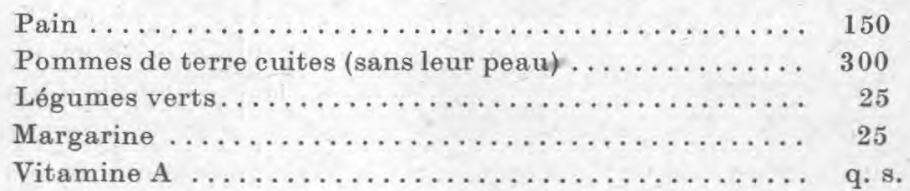

Ce régime de base, non optimal pourtant, ear assez pauvre en protides, permet une croissance et une reproduction très satisfaisantes.

L'alimentation est donnée à refus - chaque animal consommait (gaspillage inclus) de 25 à 55 grammes, suivant l'âge, de ces différents régimes par jour - eau à diserétion. Ainsi dans le régime $\mathrm{n}^{\circ}$ II, il y avait $20 \%$ en moyenne (en poids) de légumes verts.

Voici la croissance moyenne en $175-180$ jours :

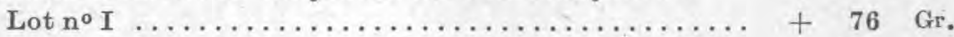

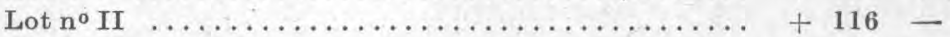

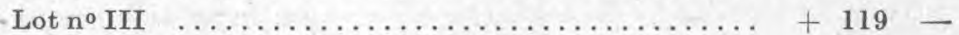

Le $175^{\mathrm{e}}$ jour (1), un dés rats du régime fromage seul est trouvé mort dans sa cage, dévoré aux trois quarts par les autres. Il n'avait, non plus que les autres animaux, présenté aucun signe clinique.

Nous sacrifions à ce moment ( $180^{\mathrm{e}}$ jour) les différents animaux. L'examen histologique ne montre aucune lésion.

Voici les résultats que nous avons éus :

\begin{tabular}{l|c|c|c|c|c|c}
\hline \hline & $\begin{array}{c}\text { Poids } \\
\text { du foie } \\
\text { (moyen.) }\end{array}$ & $\begin{array}{c}\text { Poids } \\
\text { des } \\
\text { denx reins } \\
\text { (moyen.) }\end{array}$ & $\begin{array}{c}\text { Hémo- } \\
\text { globine } \\
\text { (moyen.) } \\
\%\end{array}$ & $\begin{array}{c}\text { Globules } \\
\text { rouges } \\
\text { (moyenne) }\end{array}$ & $\begin{array}{c}\text { Globules } \\
\text { blanes }\end{array}$ & $\begin{array}{c}\text { Urée (2) } \\
\text { sanguine } \\
\text { (mélange } \\
\text { des sangs) }\end{array}$ \\
\hline Lot $\mathrm{n}^{\circ}$ I $\ldots$ & 5,59 & 1,29 & 65 & 6.400 .000 & 8.800 & 0,70 \\
Lot $\mathrm{n}^{\circ}$ II $\ldots$ & 6,47 & 1,66 & 80 & $\mathbf{7 . 0 4 0 . 0 0 0}$ & 9.000 & 0,65 \\
Lot $\mathrm{n}^{\circ}$ III . & 6,75 & 1,52 & 80 & 6.940 .000 & 14.100 & 0,55 \\
\hline \hline
\end{tabular}

(1) Rappelons que pour le rat blane (qui meurt de vieillesse vers le millième jour), dix jours de vie sont égaux à environ un an de la vie humaine.

(2) Dosage par l'hypobromite sur les animaux sacrifiés en période digestive. 
Le dosage du fer, effectué dans le laboratoire de M. Hazard, que nous remercions, nous a donné les chiffres suivants.

Pour 1.000 grammes :

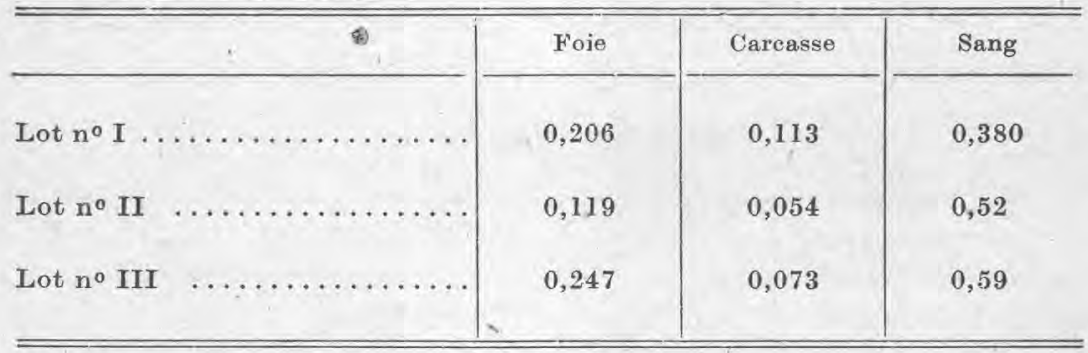

La reproduction s'était montrée la suivante (les jours sont comptés depuis la mise en expérience).

\begin{tabular}{l|c|c|c}
\hline \hline & Dates de mise bas & Nombre de petits nés & $\begin{array}{c}\text { Nombre de vivants } \\
\text { te quarantième jour }\end{array}$ \\
\cline { 2 - 3 } & Lot $\mathrm{n}^{\circ} \mathrm{I} \ldots \ldots$ & 14 & 1 (1) \\
Lot $\mathrm{n}^{\circ}$ II $\ldots . .$. & 50 et 54 jours & 16 & 9 \\
Lot $\mathrm{n}^{\circ}$ III $\ldots$ & 46 et 51 jours & 19 & 18 \\
\hline
\end{tabular}

\section{Résumons les résultats observés à la première génération}

Des animaux nourris avec le seul fromage blane, un seul est mort le $175^{\mathrm{e}}$ jour - de cause inconnue. De façon générale, ils supportent donc assez bien, pendant six mois, eette alimentation exclusive. Ils ont une croissance moindre que les animaux des deux autres lots. Ils présentent une anémie très discrète, une azotémie un peu plus élevée, peut-être en rapport avec leur surnutrition azotée, une teneur en fer discordante. Leur reproduction est un peu retardée, il y a moins de petits et ceux-ci succombent. A l'autopsie des adultes

Suite de la note de la page précédente.

Le dosage par l'hypobromite de l'urée sanguine du rat donne, nous semble-t-il, des chiffres supórieurs à ceux obtenus avec le xanthydrol, et ce n'est qu'approximatif. Nous avons abandonné cette méthode de dosage pour l'urée sanguine des rats.

(1) Il sera sacrifié, très chétif, le quarantième jour - ne présente pas de lésion apparente mais est très anémique : globules rouges, 1.640 .000 ; globules blancs, 5.200. Le poids des petits à la naissance était sensiblement comparable entre les divers lots, et cela jusqu'au dixième jour (done les fonctions de lactation et la qualité du lait paraissent normales). Mais le dixième jour les rats du lot I pesaient 10 grammes, alors que ce même dixième jour, les jeunes rats des lots II et III en pesaient 11 et -14 grammes. A partir de ce moment, il y eut d'importantes morts des petits du lot I. Nous avons laissé les petits animaux au même régime que leur mère. 
sacrifiés, pas de lésion apparente, poids du foie et du rein, par rapport au poids total, normaux.

Les animaux, nourris avec le fromage additionné de légumes verts, ont une nutrition se rapprochant de celle du lot témoin, bien que la mortalité de leurs petits soit importante.

\section{Seconde génération}

Nourris au seul fromage, les animaux de la seconde génération succombent rapidement, comme nous l'avons $v u$, en 10 à 40 jours. Par contre, au début, se développent de façon presque satisfaisante les animaux nourris de fromage, additionné d'une quantité modérée de légumes verts, nés eux-mêmes d'animaux nourris de cette façon.

Jusqu'au $70^{\mathrm{e}}$ jour, il n'y a pratiquement pas de modification apparente par rapport aux témoins. Mais à partir de ce moment, une différence devient visible, celle du poids et le $100^{\mathrm{e}}$ jour de leur naissance, le poids du lot $n^{\circ}$ II bis (descendant du lot II) est de 100 grammes en moyenne, alors que celui du lot III bis est de 145 grammes. Les animaux de chaque groupe répartis en cage (sexes mélangés) vont se comporter de façon différente.

Quatre rates témoins (les autres ayant été utilisées pour d'autres recherches) ont, du $122^{\mathrm{e}}$ au $155^{\mathrm{e}}$ jour, des petits en nombre normal (33 en tout) alors qu'aucune des rates du lot II bis ne devient gravide. Donc, suppression des fonctions de reproduction. Le $110^{\mathrm{e}}$ jour, un des animaux du lot II bis tombe pendant qu'on nettoyait sa cage et succombe quelques heures après. L'autopsie montre un œdème discret mais généralisé, cutané et séreux. L'intestin est hémorragique, le foie piqueté, les poumons congestionnés. Nous les interprétons (à tort) comme lésions secondaires à une fracture du crâne.

Mais le $140^{\circ}$ jour, un des rats du lot II bis présente un œdème considérable et généralisé. Nous le sacrifions et l'analyse montre :

\section{930.000 globules rouges \\ 17.600 globules blanes \\ $60 \%$ d'hémoglobine}

une hépatite discrète : pas de lésion rénale.

Un autre sans œdême et un quatrième avec œdème, paraissant malades, sont sacrifiés les $150^{\mathrm{e}}$ et $222^{\mathrm{e}}$ jour.

L'autopsie montre, chez l'un, une hépatite subaiguë avec foyer hémorragique - sans lésions rénales - et chez l'autre, très azotémique, (2 gr. 60) une néphrosclérose, sans albuminurie, avec légère sclérose hépatique et atrophie testiculaire (d'un seul côté).

L'examen histologique concernant ce rat hyperazotémique donne les résultats suivants : 
1. Rein. - Le rein présente des signes histologiques évidents de néphrite subaiguë glomérulo-épithéliale. En effet, la plupart des glomérules sont turgescents; les anses glomérulaires, pratiquement exsangues, sont encombrées de sérosité, de macrophages et de lipophages (les polynucléaires étant pratiquement absents). Certains glomérules, dont l'altération est plus évoluée, sont en voie de disparition par prolifération scléreuse des cellules capsulaires.

Les lésions tubulaires de ce rein sont non moins évidentes. Un nombre considérable de tubes est dilaté à l'extrême, avec une lumière obstruée de cylindres hyalins, granuleux, ou d'hématies ; la desquamation reste cependant discrète, et l'on observe surtout des images de dégénérescence vasculaire et granuleuse de l'épithélium (voire même quelques aspects de stéatose épithéliale). Il est à noter qu'en certaines zones du cortex, l'épithélium tubulaire a un aspect hypertrophique, témoin sans doute de phénomènes de compensation.

Le tissu interstitiel ne présente pratiquement pas de modifications ; il n'existe ni sclérose, ni œdème, ni congestion vasculaire ; on ne décèle que quelques discrets amas de cellules inflammatoires lympho-plasmacytaires.

2. Foie. - Le foie ne présente pas de modifications histologiques apparentes.

3. Testicule. - Il est remarquable par la diminution considérable du nombre des spermatozoïdes des adultes, s'opposant à l'intégrité presque totale des autres éléments de la lignée séminale, la densité et le volume des tubes séminifères restant normaux et le tissu interstitiel ne présentant aucune altération.

(Trois animaux nous restent, deux femelles et un mâle. Ils ne semblent ni eachectiques, ni œdémateux, mais leur poil est hérissé. On les remet au régime normal de base le $222^{\mathrm{e}}$ jour. On les laisse dans la même cage. De suite, on observe une amélioration clinique et la reproduction devient normale. Les lésions provoquées par cette alimentation paraissent done réversibles).

Le résumé de nos expériences peut ainsi s'écrire :

1. Le fromage blane donné au rat comme seul aliment permet la vie pendant six mois et les troubles paraissent, à la vérité, assez discrets. Mais si la reproduction n'est pas nulle, bien qu'atténuée, si la lactation paraît s'effectuer, la mortalité des petits nés de ces animaux et nourris de eette même façon s'avère totale.

2. Le fromage, additionné d'une quantité assez importente, de l'ordre, en poids, et en moyenne, de $20 \%$ de légumes verts, permet une croissance et une reproduction voisines de la normale bien qu'un peu moindre. Pas de morts dans cette première génération. 
3. Par contre, leurs petits alimentés toujours de la même façon, s'ils grandissent et paraissent normaux pendant 4 mois environ, ne peuvent avoir des petits et succombent avec un œdème, des lésions de néphrite avec diminution des spermatozoïdes. Ces lésions paraissent réversibles.

Ainsi, en plus de ces résultats, une notion générale se dégage, dont on ne tient pas toujours compte : une expérience alimentaire doit être poursuivie longtemps, au moins deux générations, pour qu'on puisse affirmer si tel régime est nocif ou non.

S'agissait-il de troubles de carence?

Cette opinion est tout à fait plausible. Mais par définition, le fromage, bien que relativement pauvre en fer et en cuivre, en contient pourtant des quantités non négligeables et nous n'aurions pas eu d'accidents de cet ordre dans notre lot alimenté avec fromages et légumes verts.

L'ensemble de ees deux aliments apporte, en effet, tous les éléments connus, mais il en est d'inconnus. Il y a la gamme complète des acides aminés et en proportion théoriquement (semble-t-il) satisfaisante. Il y a des acides gras non saturés. Il y a l'ensemble des vitamines connues et des sels (1).

Les troubles ne sont-ils pas, au contraire, fonction d'un déséquilibre qui, se poursuivant deux générations, finit par être nocif ? $\mathrm{Ne}$ doit-on, et cela est presque synonyme, invoquer l'intoxication par les matières protidiques en excès qui peuvent agir directement ou én modifiant la flore intestinale. Il est impossible de répondre à ces questions avec nos seules expériences.

Cependant nous pouvons les rapprocher des faits d'hépatite observés sur le rat nourri par la farine de soja cru (2). Le soja (3) a une composition en acides aminés, très analogue à celle du fromage blanc. Il est notablement plus riche en glycides ( $24 \%$ ). Il contient tous les sels et, semble-t-il, toutes les vitamines. Or, le jeune rat, ainsi alimenté, meurt rapidement : ou bien il a des troubles digestifs et se cachectise, la mort survenant en 10-25 jours; ou bien, après une phase de bonne santé, il s'effondre brusquement, succombant vers le $25^{\mathrm{e}}-40^{\mathrm{e}}$ jour. L'examen chimique décèle de l'hypoprotéinémie, de l'hypercholestérinémie, de l'albuminurie et de l'azotémie. A l'autopsie, nécrose hémorragique du foie sans surcharge ni dégénérescence graisseuse ; hypertrophie rénale, grosses capsules surré-

(1) Voir la thèse de Decourcelle sur le fromage. Paris, 1949, inspirée par l'un de nous et les travaux de $M$ me Randoin et de son école.

(2) Matet. Hépatite d'origine alimentaire : la nécrose hémorragique du foie provoquée chez le jeune rat par le soja cru. Thèse, Paris, 1949.

(3) Maupas. Le soja. Thèse, Paris, 1948. 
nales. Dégénérescence du thymus et du corps thyroïde. Reins normaux quand la mort est précoce, mais seulement dans ce cas.

La pathogénie en est mal connue. Il ne s'agit pas d'avitaminose, car la cuisson diminue ce pouvoir toxique du soja.

Comme la cystine et la méthionine améliorent l'état du rat nourri au soja cru, il est probable que, dans ce cas, il s'agit d'un trouble dans le métabolisme et dans l'équilibre des acides aminés.

\title{
LE TEST de LA PHOSPHATASE APPLIQUé A LA RECHERCHE DE LA PASTEURISATION DES LAITS DE FROMAGERIE
}

par

\author{
A. CAMUS et R. ALIFAX \\ Station centrale de Technologie agricole
}

Le rôle prépondérant joué par la flore superficielle dans la maturation des fromages et l'existence de phosphatases d'origine fongique et bactérienne nous ont conduits à rechercher l'influence des agents microbiens de la maturation sur l'épreuve de la phosphatase appliquée aux fromages.

Dans une communication précédente [1], nous avions insisté sur le fait que la phosphatase qui prend naissance dans la croûte d'un fromage semblait diffuser lentement dans la masse de celui-ci et pouvait troubler les résultats de la recherche de la pasteurisation des laits mis en œuvre pour sa fabrication.

Il nous a paru intéressant de déterminer la vitesse de cette diffusion afin d'établir les délais dans lesquels l'épreuve garde quelque valeur.

Les essais ont porté sur des fromages à pâte molle, à croûte moisie et sur des fromages à pâte pressée cuite.

\section{Essais effectués sur fromages à pâte molle à croûte moisie Camembert.}

Une série de fromages a été fabriquée à partir du lait pasteurisé d'une même cuve et ensuite traitée normalement. Le test de la phosphatase leur a été appliqué à divers stades de la fabrication. Les prélèvements ont été faits, avec toutes les précautions nécessaires pour éviter les apports de la diastase vers le centre, à la surface, à $5 \mathrm{~mm}$. de la surface, au centre du fromage. Nous avons utilisé pour le dosage de la phosphatase la méthode de Sanders et Sager [2].

Les résultats, exprimés en millièmes de milligrammes de phénol 